EDITORIAL INFORMATION 2018

Published articles

\begin{tabular}{|l|c|c|}
\hline & $\mathbf{2 0 1 7}$ & $\mathbf{2 0 1 8}$ \\
\hline Total of published articles & 16 & 15 \\
\hline Submitted to double blind review & 14 & 15 \\
\hline Invited & 2 & 0 \\
\hline Authors geographic partnership & & \\
\hline Only Brazilian authors & 5 & 10 \\
\hline Only foreigners authors & 6 & 5 \\
\hline International collaboration & 5 & 0 \\
\hline
\end{tabular}

Institutional diversity of authors (\%)

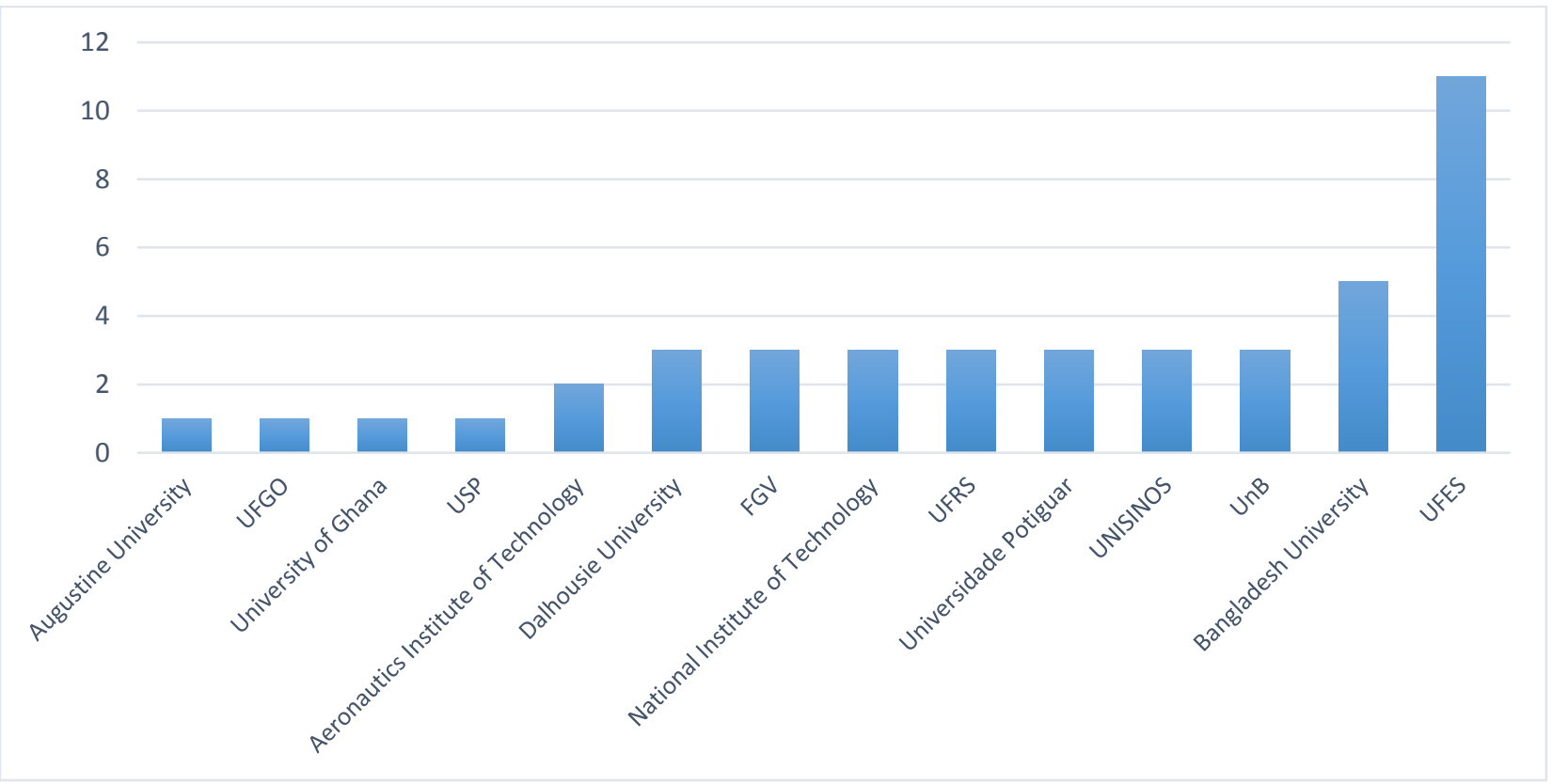

Geographic diversity of authors (\%)

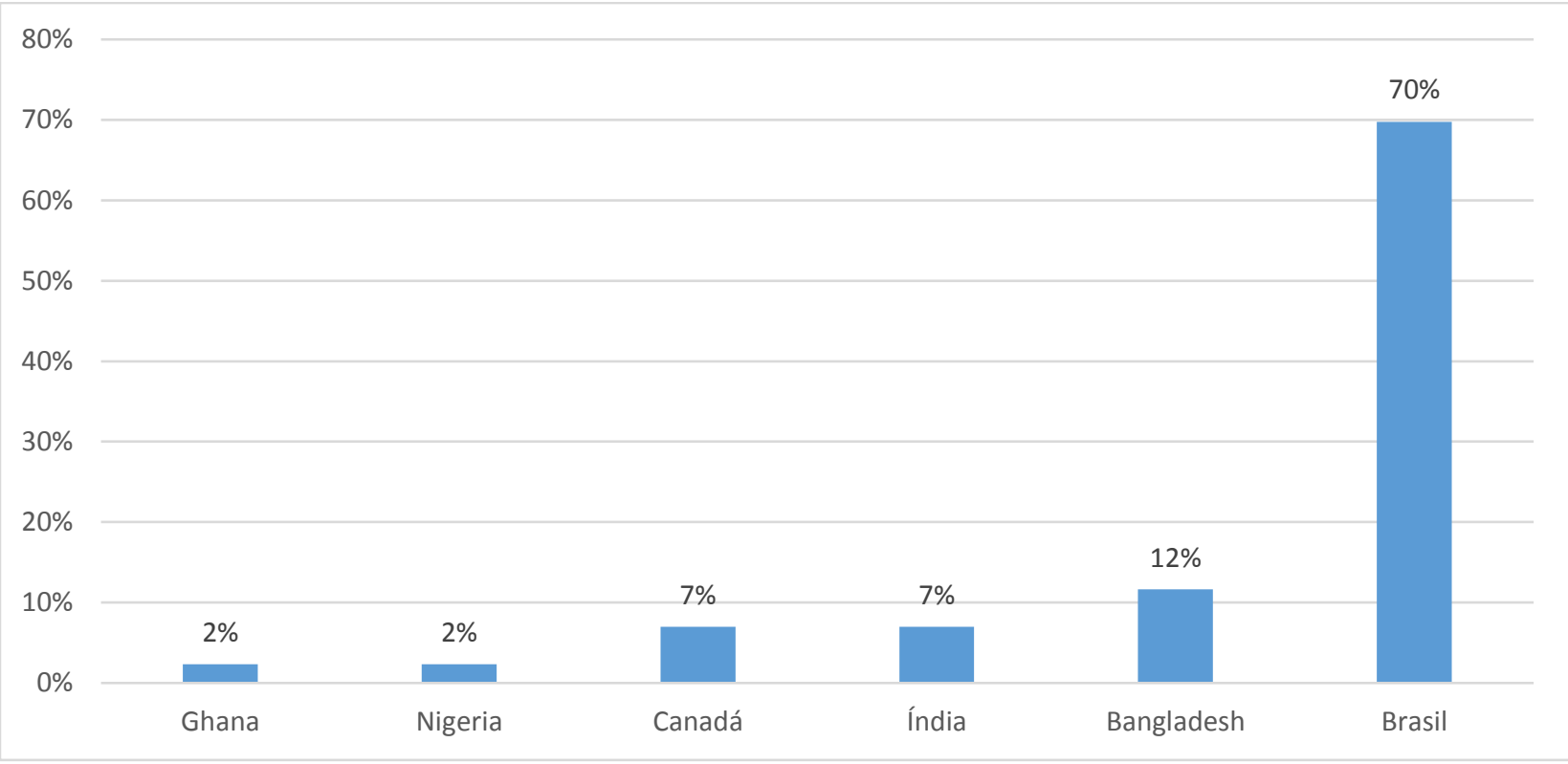




\begin{tabular}{|c|l|c|c|c|c|}
\hline \multicolumn{2}{|c|}{} & $\mathbf{2 0 1 7}$ & $\%$ & $\mathbf{2 0 1 8 1}$ & $\%$ \\
\hline AUTHORS & Submitted & $\mathbf{3 3}$ & $\mathbf{1 0 0 \%}$ & $\mathbf{2 9}$ & $\mathbf{1 0 0 \%}$ \\
\hline \multirow{2}{*}{$\begin{array}{c}\text { EDITORIAL } \\
\text { OFFICE (standard } \\
\text { review) }\end{array}$} & Non-standard & 11 & $33 \%$ & 13 & $45 \%$ \\
\cline { 2 - 6 } & Withdrawn (before the double blind review) & 1 & $3 \%$ & 0 & $0 \%$ \\
\cline { 2 - 6 } & Sent to desk review & $\mathbf{2 1}$ & $64 \%$ & $\mathbf{1 6}$ & $55 \%$ \\
\hline
\end{tabular}

\begin{tabular}{|c|l|c|c|c|c|}
\hline \multirow{2}{*}{$\begin{array}{c}\text { EDITOR (desk } \\
\text { review) }\end{array}$} & Rejected & 2 & $6 \%$ & 2 & $7 \%$ \\
\cline { 2 - 6 } & R\&R & 0 & $0 \%$ & 0 & $0 \%$ \\
\cline { 2 - 6 } & Sent to a Scientific Editor & $\mathbf{1 9}$ & $58 \%$ & $\mathbf{1 4}$ & $\mathbf{4 8 \%}$ \\
\hline
\end{tabular}

\begin{tabular}{|c|c|c|c|c|c|}
\hline \multirow{4}{*}{$\begin{array}{l}\text { SCIENTIFIC } \\
\text { EDITOR } \\
\text { (preliminary } \\
\text { review) }\end{array}$} & In review & 3 & $9 \%$ & 0 & $0 \%$ \\
\hline & Rejected & 0 & $0 \%$ & 0 & $0 \%$ \\
\hline & $R \& R$ & 0 & $0 \%$ & 0 & $0 \%$ \\
\hline & Sent to Reviewers & 16 & $48 \%$ & 14 & $48 \%$ \\
\hline
\end{tabular}

\begin{tabular}{|c|l|c|c|c|c|}
\hline \multirow{4}{*}{ Reviewers ad hoc } & In review & 3 & $9 \%$ & 0 & $0 \%$ \\
\cline { 2 - 6 } & Rejected & 3 & $9 \%$ & 6 & $21 \%$ \\
\cline { 2 - 6 } & R\&R & 0 & $0 \%$ & 0 & $0 \%$ \\
\cline { 2 - 6 } & Awaiting double blind review & 1 & $3 \%$ & 0 & $0 \%$ \\
\cline { 2 - 6 } & Sent to authors' improvement & $\mathbf{9}$ & $27 \%$ & $\mathbf{8}$ & $\mathbf{2 8 \%}$ \\
\hline
\end{tabular}

\begin{tabular}{|c|l|c|c|c|c|}
\hline \multirow{2}{*}{ AUTHORS } & In improvement & 1 & $3 \%$ & 0 & $0 \%$ \\
\cline { 2 - 6 } & Withdrawn (after the double blind review) & 0 & $0 \%$ & 1 & $3 \%$ \\
\hline $\begin{array}{c}\text { Scientific Editors } \\
\text { (revaluation) }\end{array}$ & Revaluation & 2 & $6 \%$ & 0 & $0 \%$ \\
\hline \multirow{2}{*}{ AUTHORS } & Approved & 1 & $3 \%$ & $\mathbf{0}$ & $\mathbf{0} \%$ \\
\cline { 2 - 6 } & Rejected after revaluation & $\mathbf{0}$ & $0 \%$ & 0 & $0 \%$ \\
\hline Editorial Office & Published & $\mathbf{5}$ & $15 \%$ & $\mathbf{7}$ & $\mathbf{2 4} \%$ \\
\hline
\end{tabular}

${ }^{1}$ Status on January $2^{\text {nd }} 2019$. Regarding articles submitted and processed in the period from 01.01.2018 to 12.31.2018.

Annual average of days in the evaluation process until approval

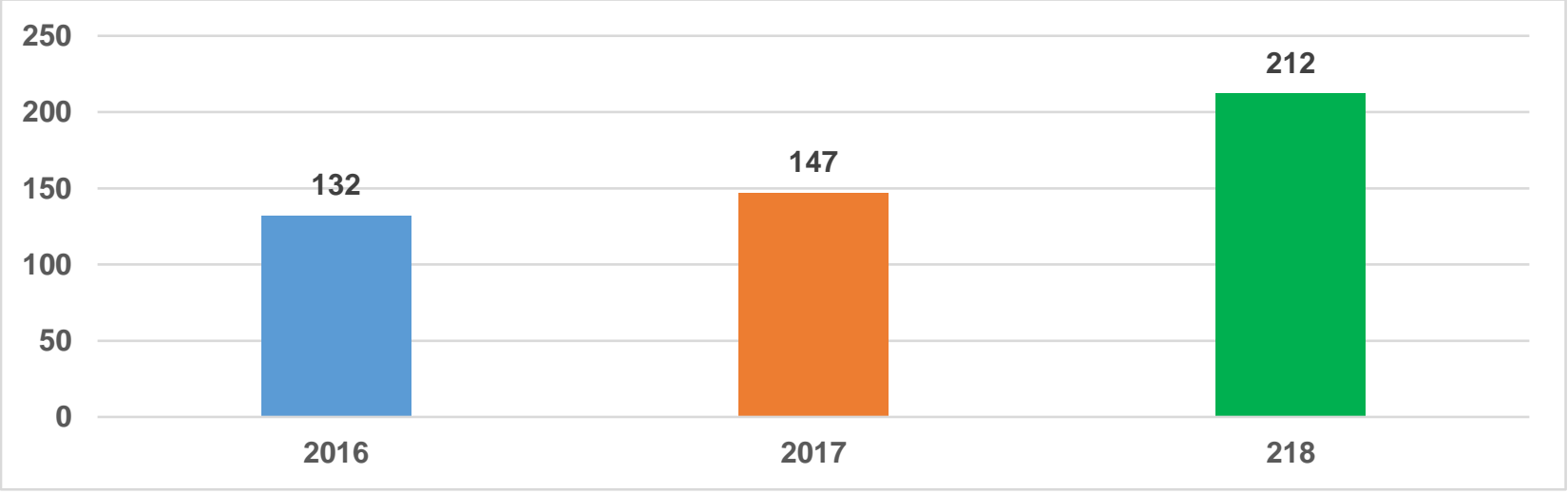




\section{REVIEWERS 2018}

The Journal of Operations and Supply Chain Management would like to thank the following reviewers for their valuable contribution:

\section{David Resende}

Universidade de Aveiro, Aveiro, Portugal

\section{Danielle Pozzo}

FADERGS, Porto Alegre, RS, Brazil

\section{Eliciane Silva}

FGV EAESP, São Paulo, SP, Brazil

Fabio Tescari

Centro Universitário Senac, São Paulo, SP, Brazil

\section{Henrique Correa}

Rollins College, Winter Park, FL, USA

\section{Jefferson Monticelli}

Universidade La Salle, Canoas, RS, Brazil

Jonas Silva

UFAM, Manaus, AM, Brazil

Juliana Santos

FGV EAESP, São Paulo, SP, Brazil

\section{Leonardo Maia}

UFU-FAGEN, Uberlândia, MG, Brazil

\section{Luciana Cezarino}

UFU-FAGEN, Uberlândia, MG, Brazil

\section{Luiz Di Serio}

FGV EAESP, São Paulo, SP, Brazil

Marcelo Sa 
FGV EAESP, São Paulo, SP, Brazil

\section{Moema Nunes}

Universidade Feevale, Novo Hamburgo, RS, Brazil

\section{Priscila Miguel}

FGV EAESP, São Paulo, SP, Brazil

\section{Roberta Souza}

USP-POLI, São Paulo, SP, Brazil

\section{Institutional diversity of reviewers}

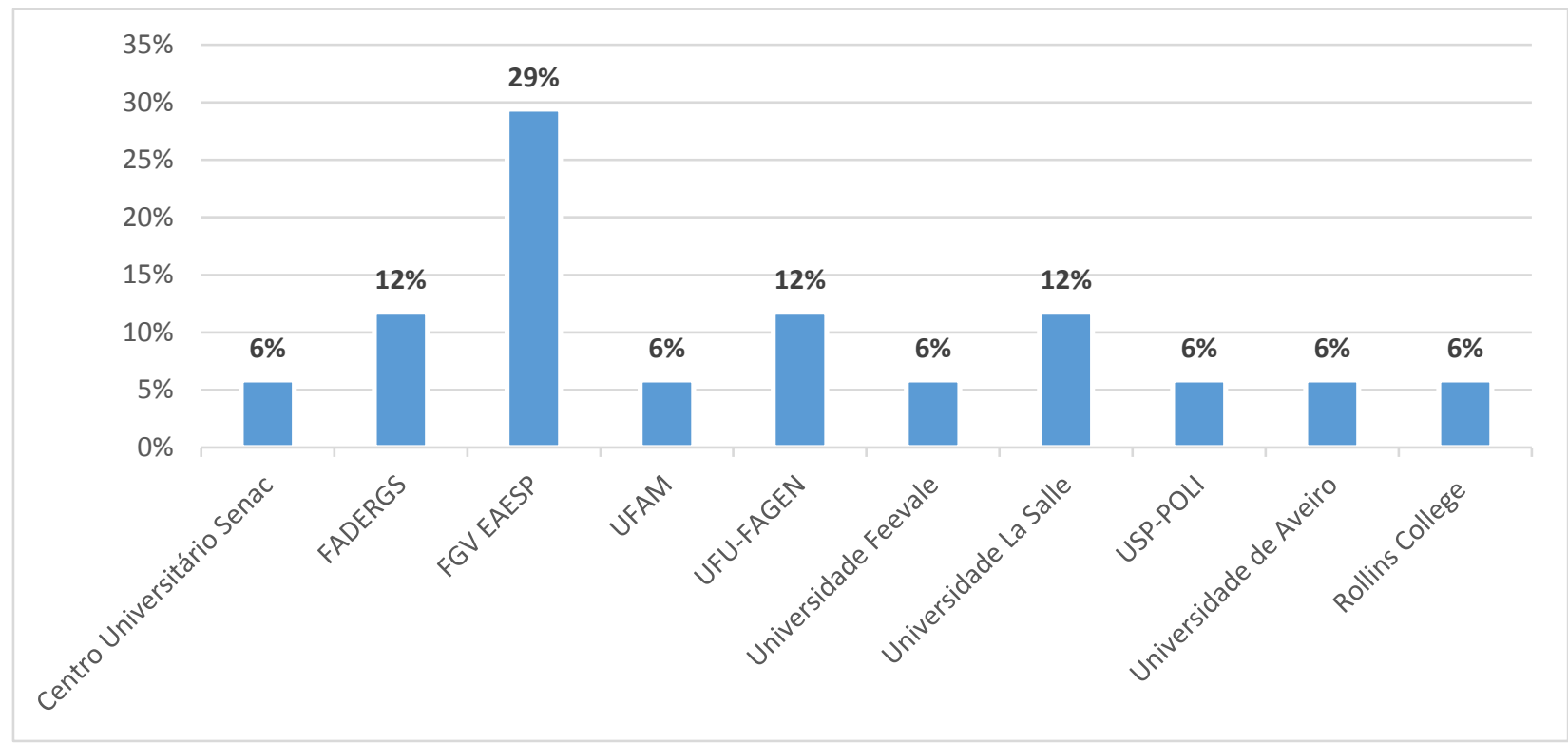

\section{Geographical diversity of reviewers}

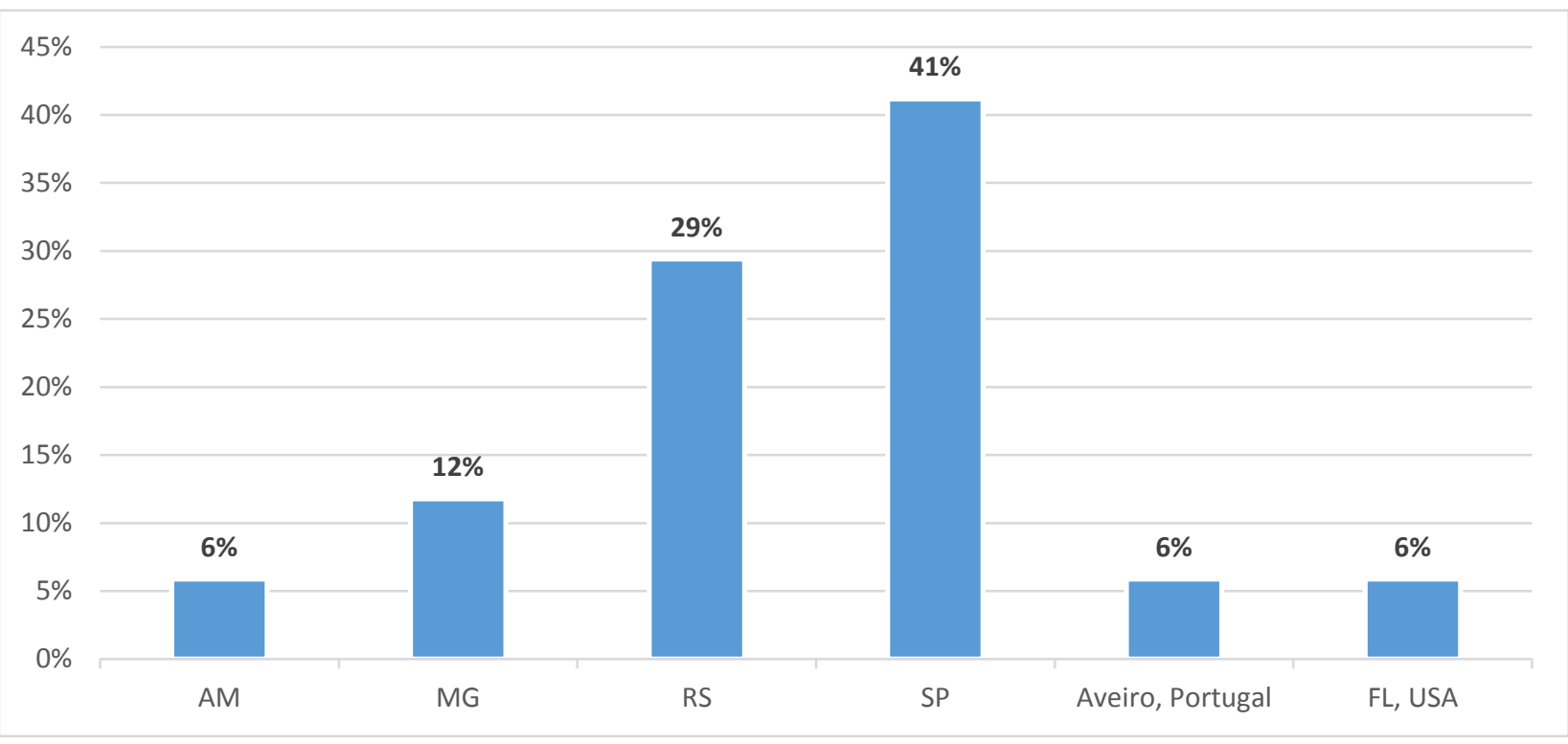

